\title{
Porous Cellulose Beads Fabricated from Regenerated Cellulose as Potential Drug Delivery Carriers
}

\author{
Lee Ken Voon, Suh Cem Pang, and Suk Fun Chin \\ Department of Chemistry, Faculty of Resource Science \& Technology, Universiti Malaysia Sarawak, 94300 Kota Samarahan, \\ Sarawak, Malaysia \\ Correspondence should be addressed to Suh Cem Pang; suhcem@gmail.com
}

Received 29 May 2017; Revised 17 August 2017; Accepted 20 August 2017; Published 26 September 2017

Academic Editor: Esteban P. Urriolabeitia

Copyright (C) 2017 Lee Ken Voon et al. This is an open access article distributed under the Creative Commons Attribution License, which permits unrestricted use, distribution, and reproduction in any medium, provided the original work is properly cited.

\begin{abstract}
Highly porous cellulose beads (CBs) of various mean sizes were successfully prepared from regenerated cellulose of paper wastes. The drug delivery characteristics of CBs with different mean sizes were investigated using curcumin as the model drug under controlled conditions. The loading capacity and efficiency of curcumin onto CBs were substantially influenced by factors such as their morphological characteristics, curcumin concentration, and duration of loading. The release kinetic profiles of curcumin from $\mathrm{CBs}$ of different mean sizes were investigated in media of $\mathrm{pH}$ values resembling digestive juices and intestinal fluids. Release kinetic models were used to simulate and elucidate release kinetics and mechanisms of curcumin from CBs under specific conditions. The loading capacity and efficiency of curcumin onto CBs could be enhanced via the optimization of curcumin solution concentration and the morphological characteristics of CBs, whereas the release kinetic profiles of curcumin from CBs could be modulated by varying the mean diameter of CBs. Optimized CBs derived from regenerated cellulose of paper wastes are potentially useful as cost-effective drug delivery carriers.
\end{abstract}

\section{Introduction}

In recent years, porous cellulose beads (CBs) derived from regenerated cellulose are receiving intense research interest for their potential biomedical and biotechnological applications such as chromatography system [1], immobilization of enzymes [2], and drug delivery system [3]. CBs are potentially cost-effective drug delivery carriers due to their high porosity and high specific surface area, favourable drug loadings, and release profiles under specific conditions [4]. Importantly, CBs are nontoxic, biocompatible, cheap, and possess excellent thermal and mechanical properties $[5,6]$.

In the pharmaceutical field, CBs constitute the main component in the formulation of drug pellets using the extrusionspheronization method [7]. CBs are prepared by mechanically shaping the wet hydrolyzed cellulose fibre fragments into spherical particles through spheronization and then loaded with model drugs such as isosorbide dinitrate, terodiline, and $\mathrm{N}$-actyl-L-cysteine for subsequent drug release studies $[8,9]$. However, the main disadvantages of the extrusionspheronization method were difficulties in controlling the size and porous structure of CBs formed which could lead to burst release instead of sustained release of drug components from the delivery system. The drug release time could be increased by loading $\mathrm{CBs}$ with paracetamol containing lipophilic release modifiers using the absorption method [10]. Different hydrophilic and hydrophobic model drugs such as anhydrous theophylline, riboflavin $5^{\prime}$-phosphate sodium, and lidocaine hydrochloride monohydrate had been incorporated into spherical CBs by the immersion method for studying their drug release profiles [3]. In addition, the effects of functionalized cellulose matrix and types of drugs on their release profiles from CBs were investigated [11, 12]. The preparation of CBs with spherical shape and mean diameter within the range of micrometer to millimeter were reported by several researchers via three main steps: dissolution of cellulose, shaping of the cellulose solution, and solidifying or precipitate the solution in a coagulation bath to form beads $[13,14]$. However, due to insolubility of cellulose fibres in water and most organic solvents, all these CBs preparation approaches required the use of derivatizing solvents and 
toxic chemicals such as carbon disulfide and epichlorohydrin, which could have adverse environmental impacts $[15,16]$.

In our previous study, we had reported a green chemistry approach for the fabrication of porous CBs from regenerated cellulose of paper wastes using nonderivatizing and environmental friendly ionic liquid as the reaction medium, and water as the antisolvent [17]. Herein, we reported the fabrication of CBs with controllable mean sizes and highly porous microstructure by varying experimental conditions including the use of extrusion syringe needle with different nozzle diameters, and different cellulose bead drying conditions. Curcumin was being used as a model drug for investigating the loading capacity and efficiency of curcumin onto CBs. The release kinetic profiles of curcumin-loaded CBs were studied in aqueous media at different $\mathrm{pH}$ values by simulating digestive juices $(\mathrm{pH}=1.23)$ and intestinal fluids $(\mathrm{pH}=6.40)$. Experimental data were subsequently being fitted to both zero and first-order kinetic equations, as well as to several empirical kinetic models for the elucidation of drug release mechanisms under specific conditions.

\section{Experiment}

2.1. Materials. Printed paper waste was used as the source of cellulose fibres. 1-Allyl-3-methylimidazolium chloride (AMIMCl), sodium dihydrogen phosphate $\left(\mathrm{NaH}_{2} \mathrm{PO}_{4}\right)$, and disodium hydrogen phosphate $\left(\mathrm{Na}_{2} \mathrm{HPO}_{4}\right)$ were purchased from Sigma Aldrich, whereas hydrochloric acid $(\mathrm{HCl})$, sodium hydroxide $(\mathrm{NaOH})$, curcumin, and sodium dodecyl sulfate (SDS) were purchased from Merck. Phosphate buffer saline solution (PBS) was prepared from 1.0 M sodium dihydrogen phosphate $\left(\mathrm{NaH}_{2} \mathrm{PO}_{4}\right)$ and $1.0 \mathrm{M}$ disodium hydrogen phosphate $\left(\mathrm{Na}_{2} \mathrm{HPO}_{4}\right)$ solutions. All chemicals were used without further purification. Ultrapure water $(\sim 18.2 \mathrm{M} \Omega \cdot \mathrm{cm}$, $25^{\circ} \mathrm{C}$ ) was obtained from the Water Purifying System (ELGA, Model Ultra Genetic).

2.2. Cellulose Fibres Extraction from Paper Wastes. $50.0 \mathrm{~g}$ of ground paper wastes was treated with $\mathrm{NaOH}$ solution $(12.0 \mathrm{wt} \%)$ for $24 \mathrm{~h}$ at room temperature in order to swell cellulose fibres, as well as to remove residual ink particles and hemicelluloses. Pretreated cellulose fibres were then immersed in $\mathrm{HCl}$ solution $(1.0 \mathrm{M})$ at $80^{\circ} \mathrm{C}$ for $2 \mathrm{~h}$ to remove lignin residuals. Purified cellulose fibres were obtained by washing thoroughly with deionized water and then dried until constant weight in an oven at $60^{\circ} \mathrm{C}$ [18]. The purity of cellulose fibres was confirmed by comparing the FTIR spectrum of CBs with that of commercial alpha-cellulose.

2.3. Preparation of Porous Cellulose Beads (CBs). CBs were prepared based on the method reported in our previous paper [17]. $10.0 \mathrm{~g}$ of $\mathrm{AMIMCl}$, an ionic liquid, was preheated at $80^{\circ} \mathrm{C}$ for $0.5 \mathrm{~h}$, and then $0.4 \mathrm{~g}$ of cellulose fibres was added into the ionic liquid and stirred at $500 \mathrm{rpm}$ for $20 \mathrm{~min}$ to obtain a cellulose solution ( $4 \mathrm{wt} \%)$. CBs were subsequently prepared by adding the cellulose solution drop-wise into an ultrapure water coagulation medium at room temperature using a syringe attached with a needle of fixed nozzle diameter.
Needles of various nozzle diameters $(1.20 \mathrm{~mm}, 0.80 \mathrm{~mm}$, and $0.50 \mathrm{~mm}$ ) were used to prepare CBs of different mean sizes. CBs formed were washed three times with ethanol and dried using a carbon dioxide supercritical point dryer (CPD-dried), in order to preserve their porous microstructure.

2.4. Sample Characterization. The surface and bulk morphology of as prepared and curcumin-loaded beads were characterized by using scanning electron microscope (JEOL JSM-6390 LA) at accelerating voltages of $5-10 \mathrm{kV}$. The mean mass and diameter of CBs were determined by measuring mass and diameter of at least 100 individual cellulose beads using an electronic balance and a microgauge, respectively. Specific surface areas $\left(S_{\mathrm{BET}}\right)$ and porosity of cellulose beads were measured using the BET Surface Area Analyzer (Quantachrome Autosorb iQ-AG) based on nitrogen gas sorption at $77 \mathrm{~K}$. The swelling ratio $(\mathrm{g} / \mathrm{g})$ of cellulose beads was determined based on method reported by Wang et al. [19].

2.5. Loading Capacity and Efficiency of Curcumin. Curcumin was loaded onto CBs using the absorption method as reported by Athira and Jyothi [20]. Curcumin was first dissolved in a given volume of absolute ethanol to form a curcumin solution of concentration $10 \mathrm{mg} / \mathrm{L}$. A given amount of CBs was then immersed in the curcumin solution and continuously stirred at $250 \mathrm{rpm}$ in darkness at room temperature for varying durations. The curcumin-loaded CBs were then removed from the curcumin solution and dried at room temperature. The amount of curcumin incorporated into CBs was determined based on methods reported by Chin et al. [21]. Curcumin-loaded CBs $(100 \mathrm{mg})$ were mechanically crushed into powder form, added into $10.0 \mathrm{~mL}$ absolute ethanol, and stirred in darkness at room temperature for $24 \mathrm{~h}$. The absorbance of resulting curcumin solution was measured using a UV-Vis spectrophotometer (Shimadzu Model UV$160 \mathrm{~A}$ ) at the wavelength of $429 \mathrm{~nm}$. A calibration curve of curcumin solution in absolute ethanol within the concentration range of $1 \mathrm{mg} / \mathrm{L}$ to $20 \mathrm{mg} / \mathrm{L}$ was used for determining curcumin loadings in CBs samples. All experiments were conducted in triplicate. The loading capacity and efficiency of curcumin for CBs were determined based on

$$
\begin{aligned}
& \text { Loading Capacity }(\mathrm{mg} / \mathrm{g}) \\
& \qquad=\frac{\text { Mass of curcumin loaded onto CBs }}{\text { Mass of CBs }} \\
& \text { Loading Efficiency }(\%) \\
& =\frac{\text { Mass of curcumin loaded onto CBs }}{\text { Mass of curcumin in solution }} \times 100 \% .
\end{aligned}
$$

2.6. Release Kinetics of Curcumin. The in vitro curcumin release from CBs was studied in media of different $\mathrm{pH}$ values simulating digestive juices (phosphate buffer, $\mathrm{pH}=1.23$ ) and intestinal fluids (phosphate buffer, $\mathrm{pH}=6.40$ ). $50 \mathrm{mg} \mathrm{CBs}$ of different mean sizes were added to $50 \mathrm{~mL}$ of phosphate buffer saline (PBS) solution containing $8 \mathrm{~g} / \mathrm{L}$ of sodium dodecyl sulfate (SDS) at $37 \pm 0.5^{\circ} \mathrm{C}$ [22], and the desired medium $\mathrm{pH}$ values were adjusted by adding diluted hydrochloric acid. 
TABLE 1: Physical properties CPD-dried porous cellulose beads CBs of different mean diameters.

\begin{tabular}{lccc}
\hline Size code & Mean diameter $(\mathrm{mm})$ & Specific surface area $\left(\mathrm{m}^{2} / \mathrm{g}\right)$ & Porosity $(\%)$ \\
\hline S1 & $0.81 \pm 0.15$ & 478 & 90.4 \\
S2 & $2.51 \pm 0.11$ & 305 & 88.9 \\
S3 & $4.60 \pm 0.09$ & 101 & 87.3 \\
\hline
\end{tabular}

At predetermined time intervals, a subsample $(1 \mathrm{~mL})$ was withdrawn and then replaced with an equal volume of buffer solution. The amount of curcumin released was determined from the corresponding absorbance values measured at the wavelength of $429 \mathrm{~nm}$ against a calibration curve of curcumin in PBS with SDS. All experiments were performed in triplicate. The $\%$ curcumin released from CBs was calculated based on

$$
\begin{aligned}
& \text { Curcumin Release (\%) } \\
& =\frac{\text { Mass of curcumin released }}{\text { Mass of curcumin in CBs }} \times 100 \% \text {. }
\end{aligned}
$$

The release of curcumin from CBs was further analyzed by fitting experimental data to various kinetic models [23, 24]. For the zero and first-order kinetics, the cumulative percentage of curcumin and the logarithmic cumulative percentage of remaining curcumin versus time (in hour) were plotted based on

$$
\begin{array}{r}
\text { Zero Order: } Q_{t}=k_{o} \times t, \\
\text { Frist Order: } \frac{\log \left(100-Q_{t}\right)}{100}=k_{1} \times t,
\end{array}
$$

where $Q_{t}$ is the cumulative percentage of curcumin released at time $t, k_{o}$ and $k_{1}$ are respective rate constants, and $t$ is time in hour.

In addition, curcumin release kinetics data were fitted to selected empirical kinetic models including the Higuchi, Hixson-Crowell, and Korsmeyer-Peppas models as shown by $[22,23]$

$$
\begin{aligned}
\text { Higuchi: } Q_{t} & =k_{H} \times t^{1 / 2}, \\
\text { Hixson-Crowell: } Q_{o}{ }^{1 / 3}-Q_{t}{ }^{1 / 3} & =k_{H R} \times t, \\
\text { Korsmever-Peppas: } \frac{M_{t}}{M_{\infty}} & =k_{K P} \times t^{n},
\end{aligned}
$$

where $Q_{t}$ is the cumulative percentage of curcumin released at time $t ; Q_{o}$ is the initial amount of the curcumin used; $k_{H}, k_{H R}$, and $k_{K P}$ are respective rate constants; $M_{t} / M_{\infty}$ is the fraction of curcumin released at time $t$; and $n$ is the release exponent which is characteristic of different release mechanisms.

\section{Results and Discussion}

3.1. Fabrication of Cellulose Beads. CBs of three different mean diameters were prepared from regenerated cellulose derived from printed paper wastes. Droplets of cellulose solution were extruded from syringe needle nozzles of varying diameter through incorporated gravity force and constant applied pressure. The mean sizes of droplets extruded were varied and controlled based on the diameter of syringe needle nozzle used. CBs were formed from droplets of cellulose solution as they were being extruded into a coagulation bath of ultrapure water. The formation of CBs could be regulated by relative diffusion rates of ultrapure water and cellulose solution into the gelling zone [13].

All CBs fabricated were observed to possess specific surface area which varied within the range of $101-478 \mathrm{~m}^{2} / \mathrm{g}$ depending on their mean diameters (Table 1). CBs with smaller mean diameter were observed to possess substantially higher specific surface area than CBs with larger mean diameters. The pore size distribution of all cellulose beads was observed to be quite similar within the range of 100-500 nm and with a mean pore diameter of about $270 \mathrm{~nm}$ (Figure 1(a)). Besides, cellulose beads of smaller mean diameter were observed to exhibit slightly higher swelling ratio than that of cellulose beads of larger mean diameter which could be attributed the slightly higher porosity of cellulose beads with smaller mean diameter. However, the swelling ratios of all beads were not affected by the $\mathrm{pH}$ of swelling medium (Figure 1(b)).

3.2. Loading of Curcumin by Cellulose Beads (CBs). As prepared dried CBs were of regular spherical shape and white in color (Figure 3(a)). Upon loading of curcumin, the CBs remained spherical in shape with similar mean diameter, but changed into bright yellow in color (Figure 3(b)). Curcumin molecules could be loaded via adsorption onto outer surfaces and/or diffusion into the porous structure of cellulose beads with interactions through the formation of hydrogen bonds (Figure 2). SEM micrographs showed distinctive differences in the microstructure of curcumin-loaded CBs and as prepared CBs. As prepared CBs were highly porous in nature with clearly visible macropores randomly distributed throughout the bulk phase. In contrast, curcumin-loaded CBs were observed to be comparatively more compact and dense with few macropores being observable in the microstructure. Most macropores of curcumin-loaded CBs could have been filled up by curcumin molecules (Figures 3(c) and 3(d)).

3.3. Effect of Curcumin Concentration. The effect of curcumin concentration on the loading capacity and associated loading 


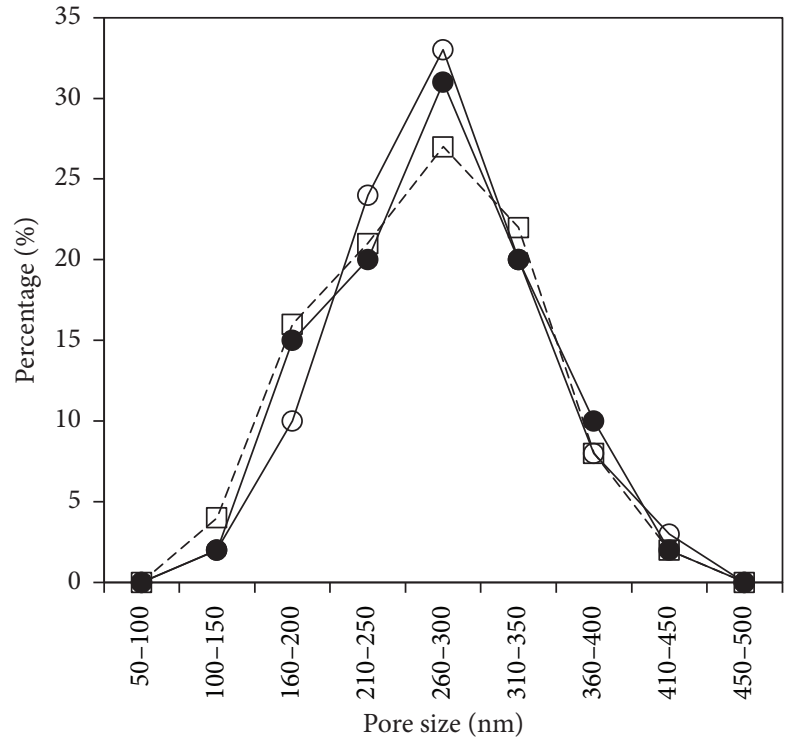

- $\square-S 1$

- $\mathrm{s} 2$

- S3

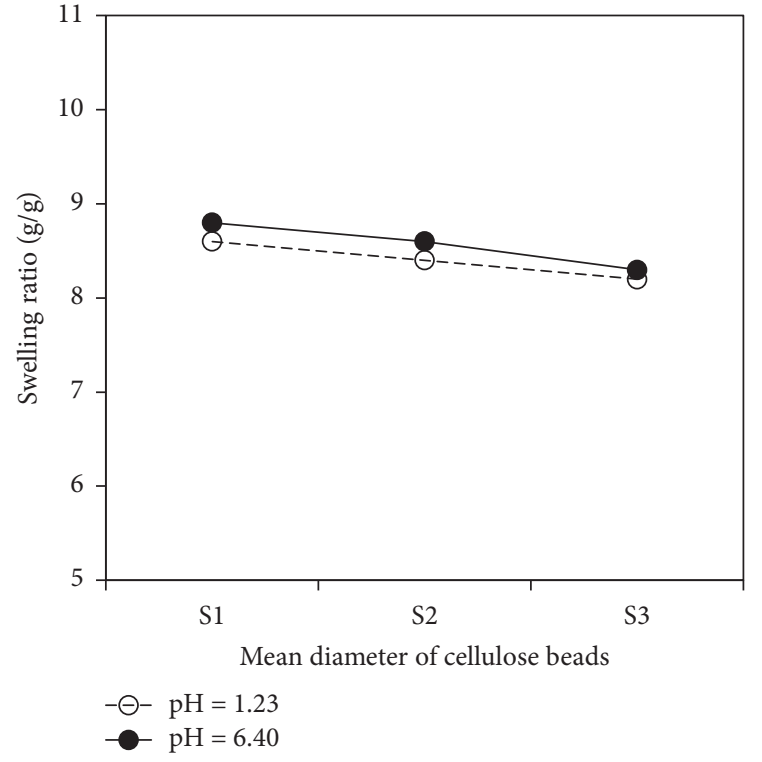

(b)

FIgURE 1: Pore size distribution (a) and (b) swelling ratio of cellulose beads with different diameter ranges.<smiles>COc1cc(/C=C/C(=O)/C=C(O)/C=C/c2ccc(O)c(OC)c2)ccc1O</smiles>

Curcumin

Cellulose beads<smiles>COc1cc(/C=C/C(=O)/C=C(O)/C=C/c2ccc(O)c(OC)c2)ccc1O</smiles><smiles>COc1cc(/C=C/C(=O)/C=C(O)/C=C/c2ccc(O)c(O)c2)ccc1O</smiles><smiles>COc1cc(/C=C/C(=O)/C=C(O)/C=C/c2ccc(O)c(OC)c2)ccc1O</smiles>

Curcumin loaded cellulose beads

FIGURE 2: Schematic representation of curcumin loaded onto cellulose beads. 


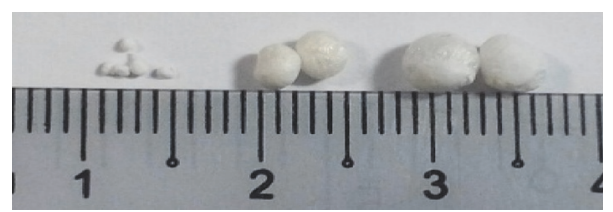

(a)

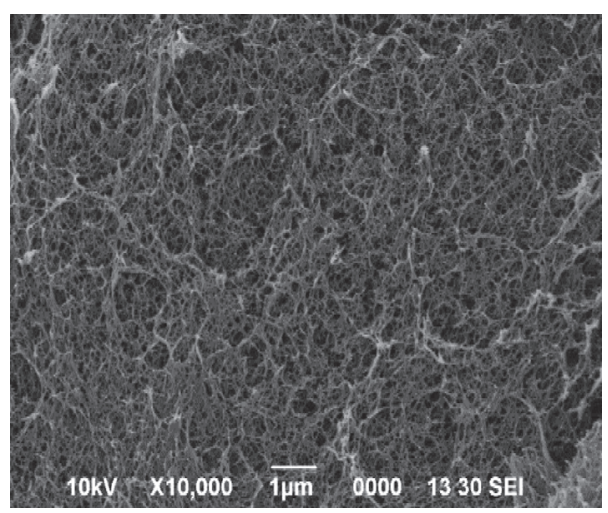

(c)

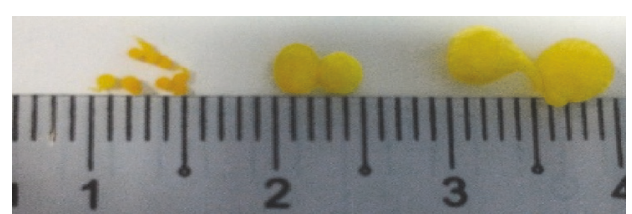

(b)

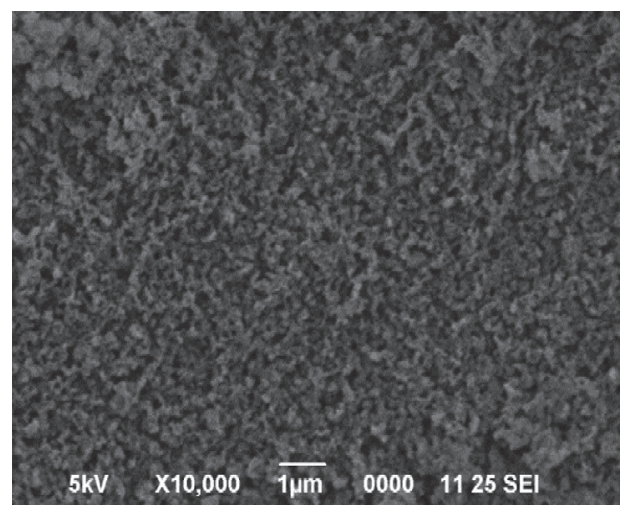

(d)

FIGURE 3: Porous cellulose beads (CBs) of different mean sizes derived from printed paper wastes: (a) As prepared; S1, S2, and S3 (left to right), (b) curcumin-loaded CBs; S1, S2, and S3 (left to right); SEM micrographs of cross sectional view for (c) as prepared CBs (S1), and (d) curcumin-loaded CBs (S1).

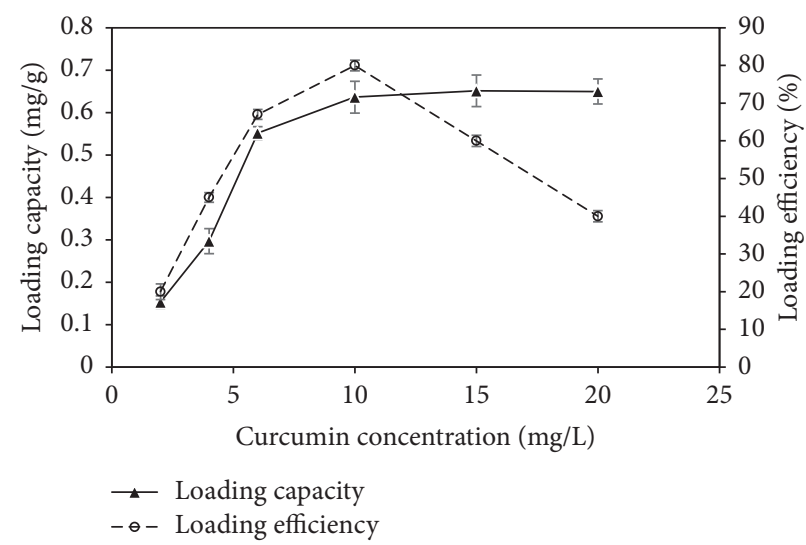

FIGURE 4: The effect of curcumin solution concentration on the loading capacity and loading efficiency of curcumin for CPD-dried CBs (S2).

efficiency of curcumin for CPD-dried CBs (S2) was shown in Figure 4. The concentration of curcumin solution was observed to have substantial effect on both the loading capacity and the loading efficiency of curcumin which were observed to increase rapidly initially with increase in the concentration of curcumin solution within the range of 2 to $6 \mathrm{mg} / \mathrm{L}$. However, the increase in loading capacity and efficiency of curcumin was observed to level off gradually at concentration range between $6 \mathrm{mg} / \mathrm{L}$ and $10 \mathrm{mg} / \mathrm{L}$. At curcumin solution concentration of $10 \mathrm{mg} / \mathrm{L}$ and above, the loading capacity of curcumin had remained constant, whereas the associated loading efficiency of curcumin was observed to have decreased substantially, indicating that the loading capacity of curcumin for CPD-dried CBs (S2) had been reached. In this study, the loading capacity and maximum loading efficiency of curcumin for CPD-dried CBs (S2) were determined to be $0.65 \mathrm{mg} / \mathrm{g}$ and $80 \%$, respectively.

3.4. Morphological Effects. The morphological effects of CBs on their loading capacity of curcumin were investigated using CBs prepared by different drying techniques: CPD-, air-, and oven-dried CBs. Figures 5(a)-5(c) show SEM micrographs of CBs prepared by different drying techniques. CPD-dried CBs showed distinctively different surface morphology with highly porous microstructures as compared with those of air-dried or oven-dried CBs which exhibited much more compact microstructure probably due to collapse of pores during the respective drying processes. Evidently, the BET 


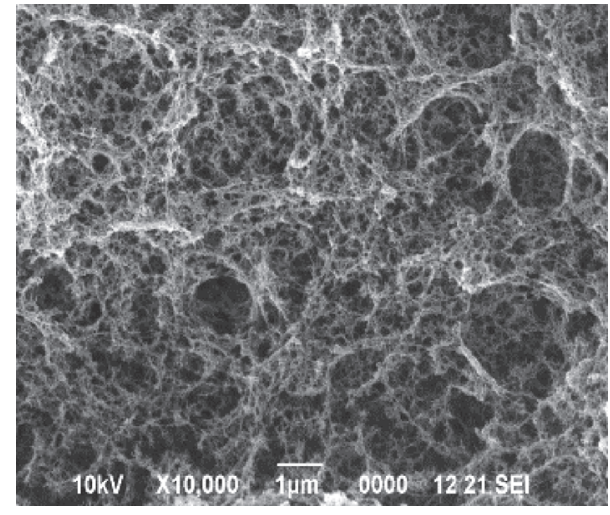

(a)

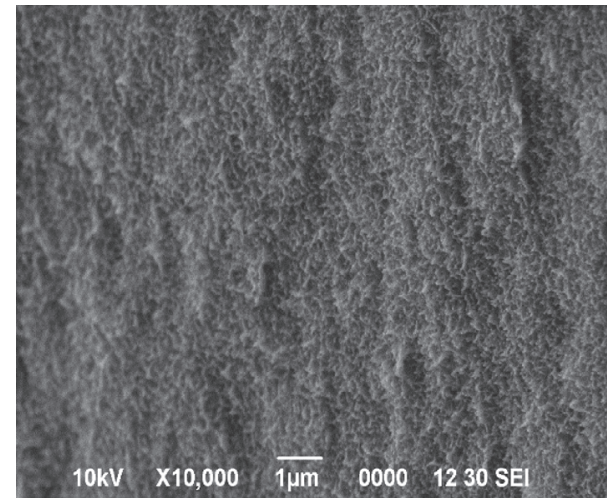

(c)

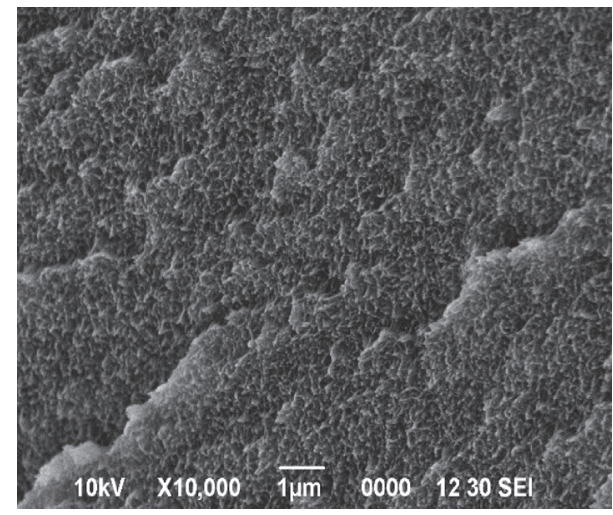

(b)

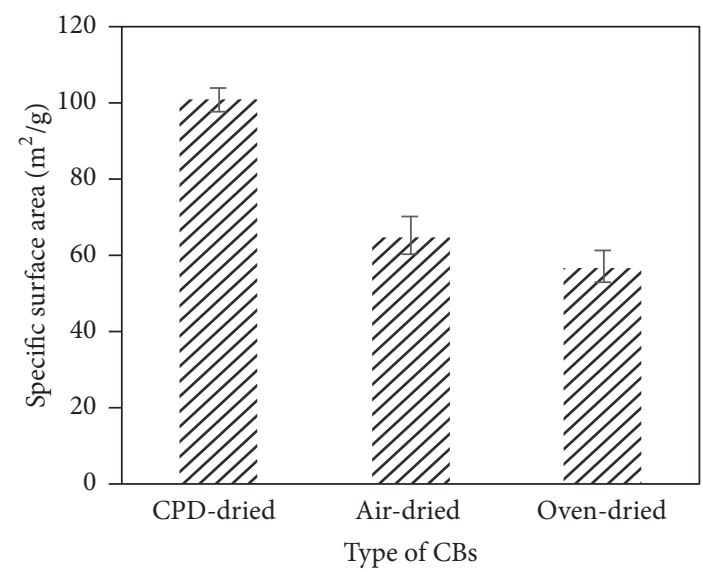

(d)

FIGURE 5: SEM micrographs of CBs prepared using different drying techniques (a) CPD-dried, (b) air-dried at room temperature, (c) ovendried at $60^{\circ} \mathrm{C}$, and (d) BET specific surface areas of CBs prepared using different drying techniques.

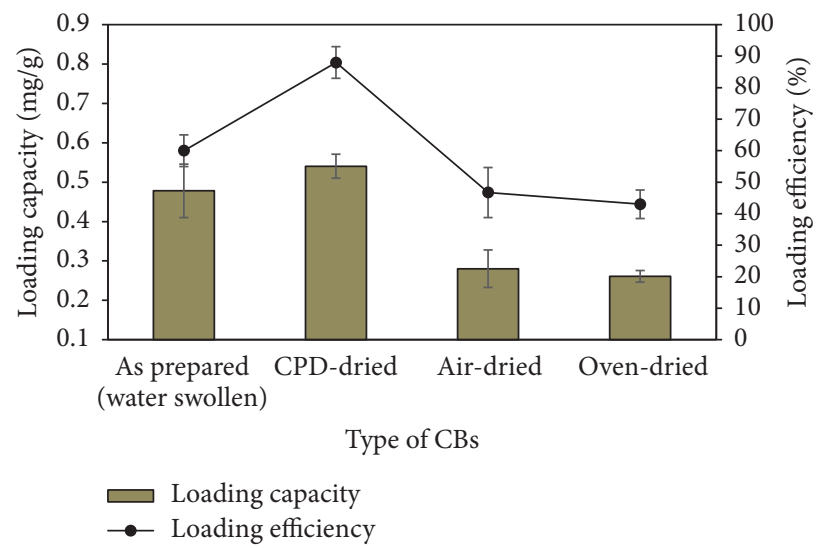

FIGURE 6: The loading capacity and efficiency of curcumin for CBs (S3) as prepared (in water swollen state), and prepared using different drying techniques.

specific surface area of CPD-dried CBs was substantially higher at $105 \mathrm{~m}^{2} / \mathrm{g}$ as compared to those of air- and ovendried CBs at $62 \mathrm{~m}^{2} / \mathrm{g}$ and $57 \mathrm{~m}^{2} / \mathrm{g}$, respectively (Figure 5(d)).

Figure 6 shows the effect of various drying conditions on the loading capacity and efficiency of CBs. CPD-dried CBs exhibited substantially higher loading capacity and efficiency of curcumin $(0.55 \mathrm{mg} / \mathrm{g} ; 88 \%)$ as compared with those of airdried CBs $(0.27 \mathrm{mg} / \mathrm{g} ; 46 \%)$ and oven-dried CBs $(0.26 \mathrm{mg} / \mathrm{g}$; $43 \%)$. The substantially enhanced loading capacity and efficiency of curcumin for CPD-dried CBs could be attributed to the formation of highly porous nanofibrillar networks with interconnected open macropores. The high porosity of CPDdried CBs enabled easy penetration by curcumin molecules 


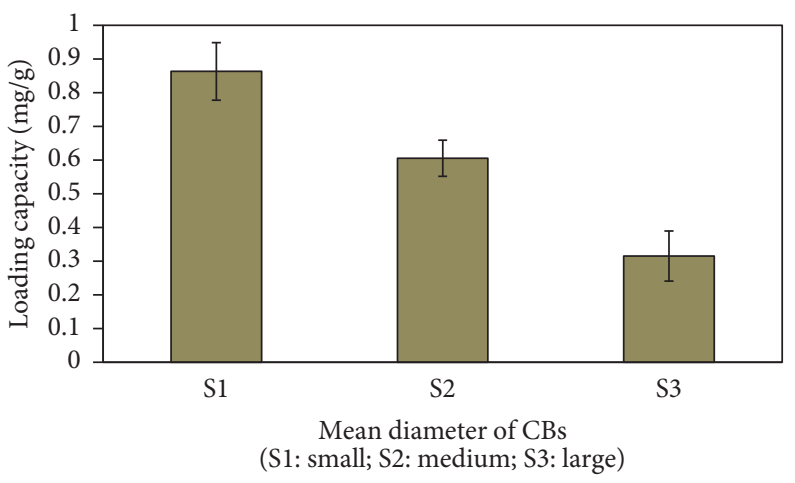

FIGURE 7: Effect of bead size on the loading capacity of curcumin for CPD-dried CBs with mean diameter of CBs $(\mathrm{S} 1=0.81 \pm 0.15 \mathrm{~mm}$; $\mathrm{S} 2=$ $2.51 \pm 0.11 \mathrm{~mm} ; \mathrm{S} 3=4.60 \pm 0.09 \mathrm{~mm}$ ).

in solution, thereby contributing towards the observed comparatively higher loading capacity and efficiency. In the case of both air-dried and oven-dried CBs, loading of curcumin molecules could be confined to adsorption onto surfaces of beads with limited penetration into the compact and densely packed microstructure of CBs, and hence substantially lower loading capacity and loading efficiency of curcumin were observed.

In the case of as prepared water swollen CBs, comparatively higher loading and loading efficiency $(0.47 \mathrm{mg} / \mathrm{g}$; $61 \%)$ than both of air- and oven-dried CBs were observed. This could be attributed to diffusion driven processes which occurred continuously until an equilibrium state was established between curcumin solution and adsorbed water within the CBs.

3.5. Effect of Mean Bead Diameter. The effect of mean bead diameter on the loading capacity of curcumin was investigated for CBs of three different mean diameters (Table 1). As shown in Figure 7, the loading capacity and efficiency of curcumin were substantially affected by the mean diameter of CBs. CBs of the smallest mean diameter $(0.81 \pm$ $0.15 \mathrm{~mm}$ ) exhibited the highest loading capacity of curcumin $(0.86 \mathrm{mg} / \mathrm{g})$, whereas CBs of larger mean diameters $(2.51 \pm$ $0.11 \mathrm{~mm}$ and $4.60 \pm 0.09 \mathrm{~mm}$ ) showed comparatively lower loading capacity of curcumin at $0.61 \mathrm{mg} / \mathrm{g}$ and $0.32 \mathrm{mg} / \mathrm{g}$, respectively. This could be attributed to the longer diffusion pathways and lower specific surface area accessible during loading of curcumin onto CBs of larger mean diameters. As reported in our recent paper, the BET specific surface area of cellulose beads was observed to vary inversely with their mean diameter [17]. Lewis et al. [25] also reported that higher loading capacities of drugs were observed for sulfonic acid groups functionalized poly(vinyl alcohol) beads of smaller mean sizes.

3.6. Loading of Curcumin. The loading of curcumin onto CBs was conducted by dispersing a known amount of CBs in a given volume of ethanolic curcumin solution of known concentration upon incubation at room temperature and in darkness for various predetermined durations. Figure 8(a) shows the UV-Vis absorption spectra of ethanolic solution of curcumin from crushed samples of CBs that had been incubated for different durations within the wavelength range of $350 \mathrm{~nm}$ and $500 \mathrm{~nm}$. The absorbance value of the characteristic absorption peak at the wavelength of $429 \mathrm{~nm}$ was observed to increase with incubation duration, indicating increasing amount of curcumin being loaded onto the CBs.

As shown in Figure 8(b), the loading of curcumin was observed to increase very rapidly initially between incubation duration at 0 and $12 \mathrm{~h}$, but thereafter slowed down gradually between 12 and $24 \mathrm{~h}$, and eventually leveled off after incubation for $36 \mathrm{~h}$. Hence, a loading capacity of curcumin at $0.52 \mathrm{mg} / \mathrm{g}$ CBs was achieved upon incubation for about $36 \mathrm{~h}$.

3.7. Release Kinetics of Curcumin. The release kinetics of curcumin from CBs of different mean sizes was investigated in media of $\mathrm{pH} 1.23$ and $\mathrm{pH} 6.40$ as simulated digestive juices and intestinal fluids, respectively (Figure 9). The release kinetic profiles of curcumin at both media $\mathrm{pH}$ showed that curcumin was being released at faster rates for CBs with decreasing mean sizes (i.e., \% curcumin released S1 > S2 > S3). Such difference was more predominant at medium $\mathrm{pH}$ 1.23 as compared with that at medium $\mathrm{pH}$ 6.40. At medium $\mathrm{pH}$ of $1.23,50.1 \%, 30.2 \%$, and $17.3 \%$ of curcumin were released within the initial $1 \mathrm{~h}$, whereas up to $95.1 \%, 73.7 \%$, and $54.3 \%$ of curcumin were released within $10 \mathrm{~h}$ from S1, S2, and S3 beads, respectively, (Figure 9(a)). The enhanced curcumin release rate from beads of smaller mean sizes could be attributed to their larger specific surface area to volume ratios, and shorter diffusion pathways which collectively enabled rapid diffusion of PBS solution into the CBs. The resulting swollen beads would enable rapid release of curcumin from the bulk phase of CBs. However, the $\mathrm{pH}$ of medium was observed to have minimal effect on the release rate of curcumin from CBs which could be attributed to their similar swelling ratios of $\mathrm{CBs}$ in different $\mathrm{pH}$ medium (Figure 1(b)).

The release kinetics and mechanisms of curcumin for CBs of different mean sizes were further evaluated by fitting to both zero and first-order kinetic equations, as well as various empirical kinetics models, namely, the HixsonCrowell, Higuchi, and Korsmeyer-Peppas models. All kinetic 


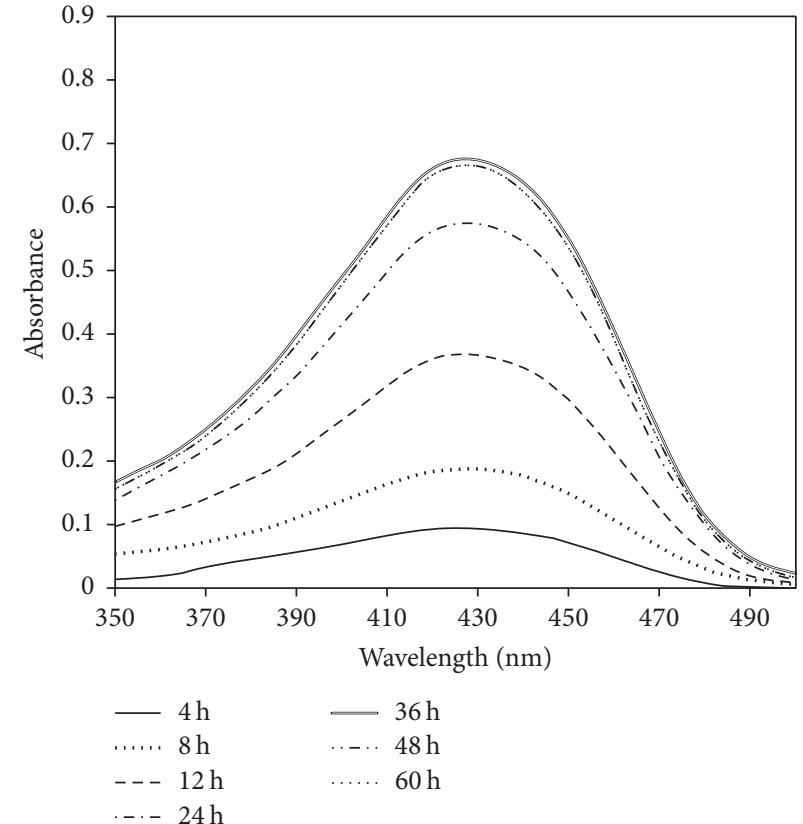

(a)

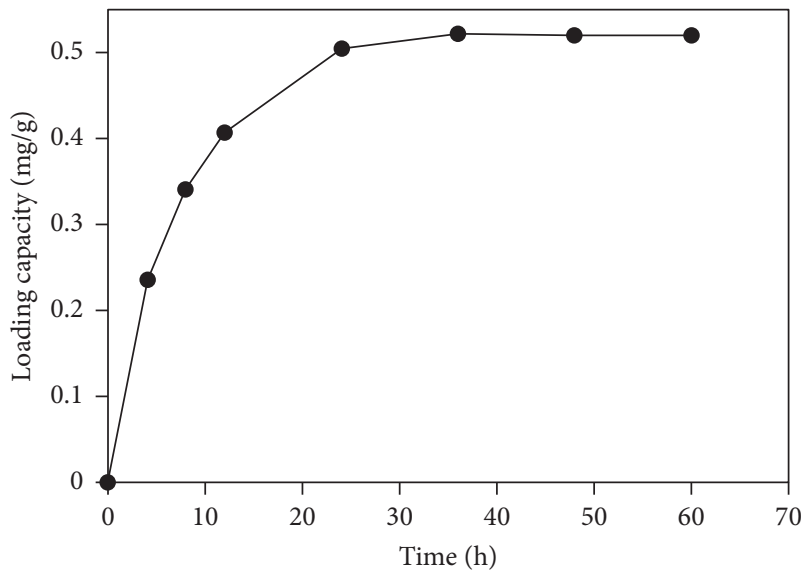

(b)

FIGURE 8: Effect of incubation duration on the curcumin loading of CBs (a) UV-Vis spectra of curcumin, (b) loading capacity of curcuminloaded CBs.

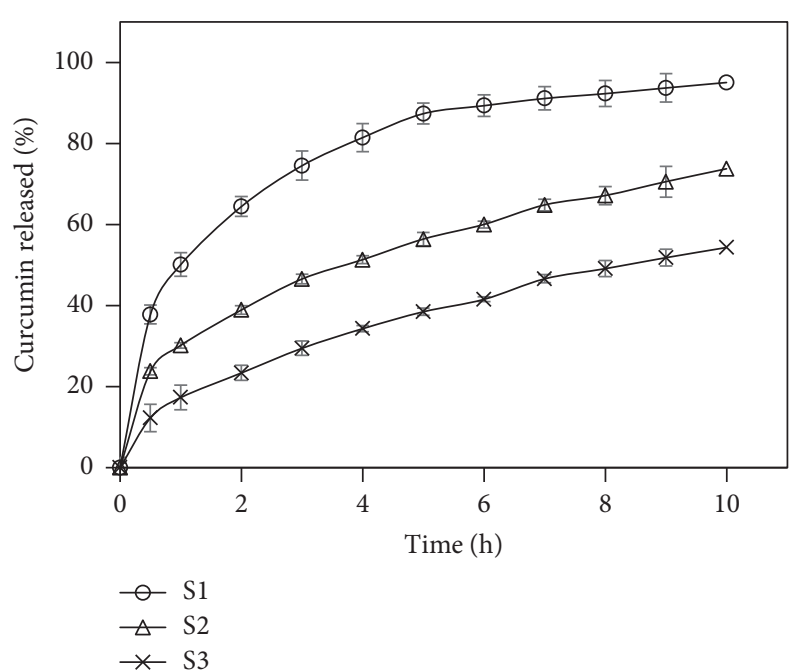

(a)

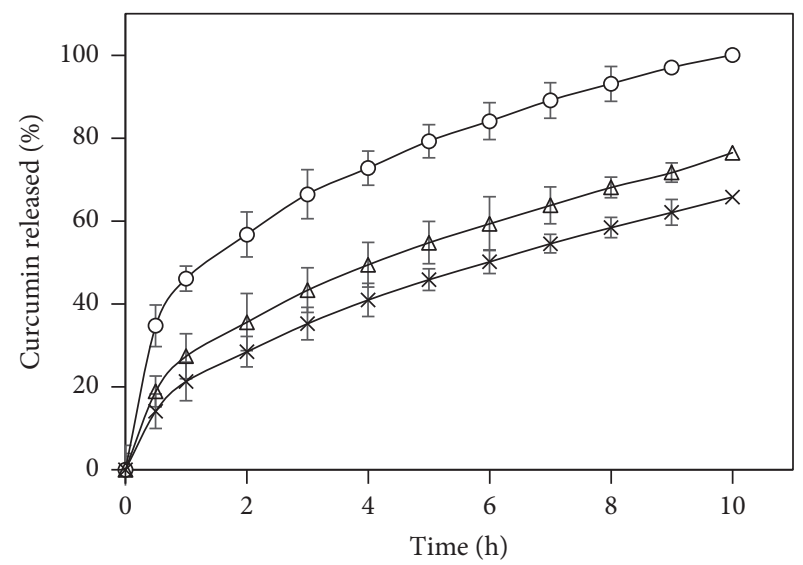

$$
\begin{aligned}
& -\mathrm{S} 1 \\
& \triangle \mathrm{S} 2 \\
& \times \mathrm{S} 3
\end{aligned}
$$

(b)

FIgURE 9: Release kinetic profiles of curcumin for CBs with different mean sizes in PBS solution at (a) pH 1.23 and (b) pH 6.40.

parameters for the release of curcumin from CBs of different mean sizes and at different media $\mathrm{pH}$ values are presented in Table 2 .

The release kinetics of curcumin for CBs was observed to conform better to the first-order kinetic equation without prominent effects by both bead mean size and medium $\mathrm{pH}$ value. $\mathrm{CBs}$ of different mean sizes exhibited regression coefficient values of 0.99 , indicating that the rate of curcumin release from CBs was mainly dependent upon the total loading of curcumin within CBs. The release of drug molecules from a polymeric substrate could be controlled though three important mechanisms which included swelling followed by diffusion, erosion, and diffusion [26]. According to the Higuchi kinetic model, drug release was based on the Fickian diffusion from swellable insoluble polymer, whereas the Hixson-Crowell model correlated drug release through erosion or dissolution of bead surfaces [27]. The release of curcumin from CBs of different mean sizes and at different media $\mathrm{pH}$ values showed considerably better fit to the Higuchi model $\left(R^{2} \sim 0.99\right)$ than the Hixson and Crowell 
TABLE 2: Released kinetics parameters for curcumin from CBs in media of different $\mathrm{pH}$ values.

\begin{tabular}{|c|c|c|c|c|c|c|c|}
\hline \multirow{3}{*}{ Kinetic model } & \multirow{3}{*}{ Bead size } & \multicolumn{6}{|c|}{ Medium $\mathrm{pH}$} \\
\hline & & \multicolumn{3}{|c|}{$\mathrm{pH}=1.2$} & \multicolumn{3}{|c|}{$\mathrm{pH}=6.4$} \\
\hline & & $R^{2}$ & \multicolumn{2}{|r|}{$k_{0}\left(h^{-1}\right)$} & $R^{2}$ & \multicolumn{2}{|r|}{$k_{0}\left(h^{-1}\right)$} \\
\hline \multirow{3}{*}{ Zero order } & S1 & 0.8134 & \multicolumn{2}{|r|}{5.3761} & 0.9478 & \multicolumn{2}{|r|}{7.0722} \\
\hline & S2 & 0.9561 & \multicolumn{2}{|r|}{5.0045} & 0.9684 & \multicolumn{2}{|r|}{5.6761} \\
\hline & S3 & 0.9722 & \multicolumn{2}{|r|}{4.3256} & 0.9740 & \multicolumn{2}{|r|}{5.1764} \\
\hline \multirow{4}{*}{ First Oder } & & $R^{2}$ & \multicolumn{2}{|r|}{$k_{1}\left(h^{-1}\right)$} & $R^{2}$ & \multicolumn{2}{|r|}{$k_{1}\left(h^{-1}\right)$} \\
\hline & S1 & 0.9769 & \multicolumn{2}{|r|}{-0.1138} & 0.9662 & \multicolumn{2}{|r|}{-0.1460} \\
\hline & S2 & 0.9950 & \multicolumn{2}{|r|}{-0.0470} & 0.9966 & \multicolumn{2}{|r|}{-0.0532} \\
\hline & S3 & 0.9934 & \multicolumn{2}{|r|}{-0.0295} & 0.9979 & \multicolumn{2}{|r|}{0.0404} \\
\hline \multirow{4}{*}{ Higuchi } & & $R^{2}$ & \multicolumn{2}{|r|}{$k_{H}\left(h^{1 / 2}\right)$} & $R^{2}$ & \multicolumn{2}{|r|}{$k_{H}\left(h^{1 / 2}\right)$} \\
\hline & S1 & 0.9274 & \multicolumn{2}{|r|}{22.795} & 0.9958 & \multicolumn{2}{|r|}{27.470} \\
\hline & S2 & 0.9983 & \multicolumn{2}{|r|}{20.307} & 0.9993 & \multicolumn{2}{|r|}{22.897} \\
\hline & S3 & 0.9987 & & 0.9994 & \multicolumn{2}{|r|}{20.823} \\
\hline & & $R^{2}$ & \multicolumn{2}{|r|}{$k_{H C}\left(h^{-1}\right)$} & $R^{2}$ & \multicolumn{2}{|r|}{$k_{H C}\left(h^{-1}\right)$} \\
\hline & S1 & 0.7613 & & -0.0172 & 0.8987 & & -0.1420 \\
\hline Hixson and Crowell & S2 & 0.9064 & & -0.1265 & 0.9098 & & -0.1480 \\
\hline & S3 & 0.9159 & & -0.1440 & 0.9129 & & -0.1530 \\
\hline & & $R^{2}$ & $n$ & $k_{K P}\left(h^{-n}\right)$ & $R^{2}$ & $n$ & $k_{K P}\left(h^{-n}\right)$ \\
\hline & S1 & 0.9716 & 0.31 & 1.7008 & 0.9990 & 0.35 & 1.6659 \\
\hline $\begin{array}{l}\text { Korsmeyer- } \\
\text { Peppas }\end{array}$ & $\mathrm{S} 2$ & 0.9992 & 0.38 & 1.4834 & 0.9988 & 0.45 & 1.4205 \\
\hline & S3 & 0.9989 & 0.50 & 1.2331 & 0.9989 & 0.51 & 1.3093 \\
\hline
\end{tabular}

model $\left(R^{2}=0.76-0.92\right)($ Table 2), indicating that the release of curcumin could be mainly attributed to the Fickian diffusion process from swollen CBs. In order to further confirm the mechanism of curcumin release, the experimental data was fitted using the Korsmeyer-Peppas model [27]. The value of release exponent, $(n)$ was indicative of the drug release mechanism. In this study, CBs of three different mean sizes showed good fit to the Korsmeyer-Peppas model $\left(R^{2}>0.97\right)$ in releasing media of different $\mathrm{pH}$ values (1.23 and 6.40). The release exponent $(n)$ values for $\mathrm{S} 1$ beads in media $\mathrm{pH}$ values of 1.23 and 6.40 were determined to range between 0.31 and 0.35 , which were beyond the limits of the Korsmeyer model, indicating that the release of curcumin could be attributed to complex mechanisms of swelling, diffusion, and erosion. Shoaib et al. [28] reported similar result on release kinetics of ibuprofen from hydroxypropyl methylcellulose. S2 beads showed similar complex release mechanism as S1 beads in medium of $\mathrm{pH} 1.23$ but exhibited rather different release mechanism in medium $\mathrm{pH}$ 6.4. The release exponent $(n)$ value for S2 beads in medium of $\mathrm{pH} 6.4$ was 0.45 indicating the release mechanism was based on non-Fickian diffusion. The release exponent $\mathrm{n}$ values for $\mathrm{S} 3$ beads were 0.50 and 0.51 in both medium $\mathrm{pH}$ values, indicating that the release mechanism could be attributed mainly to non-Fickian diffusion with molecular diffusion of drug occurred through chemical potential gradient [29]. These different release mechanisms could have contributed towards the observed differences in the release kinetic profiles of curcumin from CBs of different mean diameters.

\section{Conclusion}

Highly porous cellulose beads of different mean sizes were prepared from regenerated cellulose of paper wastes via a facile and green process. These cellulose beads were subsequently loaded with a model drug, curcumin, under specific experimental conditions. The loading capacity and loading efficiency of curcumin for CBs were prominently affected by their morphological characteristics, notably porosity, specific surface area, and mean diameter. CPD-dried CBs exhibited the highest loading capacity of curcumin at $0.86 \mathrm{mg} / \mathrm{g}$ with a loading efficiency exceeding $80 \%$. The release kinetic profiles of curcumin for CPD-dried CBs were substantially affected by the bead mean diameter, and to a lesser extent the $\mathrm{pH}$ of releasing medium. Fitting of empirical kinetic models had led to the elucidation of a Fickian diffusion controlled process as the main underlying release mechanisms of curcumin for CPD-dried CBs. The loading capacity and efficiency of curcumin for CBs could be modulated and enhanced via the optimization of curcumin solution concentration and the morphological characteristics of CBs, whereas the release kinetic profiles of curcumin could be modulated by varying the mean diameter of CBs. The potential utility of optimized CBs as cost-effective drug deliver carriers derived from regenerated cellulose via facile and green processes is therefore envisaged.

\section{Conflicts of Interest}

The authors declare that they have no conflicts of interest. 


\section{Acknowledgments}

The authors wish to acknowledge the financial support rendered by the Ministry of Higher Education, Malaysia, via the award of fundamental research grants (Grant nos. FRGS/ST01(01)/967/2013(08) and F07/FRGS/1495/2016), as well as research management and support services provided by the Research Innovation and Management Center (RIMC), Universiti Malaysia Sarawak. The authors also wish to acknowledge Professor Dr. Colin L. Raston, School of Chemical and Physical Sciences, Flinders University, Australia, for his suggestions and comments on the manuscript.

\section{References}

[1] K.-F. Du, M. Yan, Q.-Y. Wang, and H. Song, "Preparation and characterization of novel macroporous cellulose beads regenerated from ionic liquid for fast chromatography," Journal of Chromatography A, vol. 1217, no. 8, pp. 1298-1304, 2010.

[2] S. Park, S. H. Kim, J. H. Kim et al., "Application of cellulose/lignin hydrogel beads as novel supports for immobilizing lipase," Journal of Molecular Catalysis B: Enzymatic, vol. 119, pp. 33-39, 2015.

[3] E. Yildir, R. Kolakovic, N. Genina et al., "Tailored beads made of dissolved cellulose - Investigation of their drug release properties," International Journal of Pharmaceutics, vol. 456, no. 2, pp. 417-423, 2013.

[4] J. Trygg, P. Fardim, M. Gericke, E. Mäkilä, and J. Salonen, "Physicochemical design of the morphology and ultrastructure of cellulose beads," Carbohydrate Polymers, vol. 93, no. 1, pp. 291-299, 2013.

[5] K. Thümmler, S. Fischer, A. Feldner et al., "Preparation and characterization of cellulose microspheres," Cellulose, vol. 18, no. 1, pp. 135-142, 2011.

[6] A. Nussinovitch, "Bead formation, strengthening, and modification," Polymer Macro- and Micro-Gel Beads: Fundamentals and Applications, pp. 1-303, 2010.

[7] J. C. Wlosnewski, M. Kumpugdee-Vollrath, and P. Sriamornsak, "Effect of drying technique and disintegrant on physical properties and drug release behavior of microcrystalline cellulosebased pellets prepared by extrusion/spheronization," Chemical Engineering Research and Design, vol. 88, no. 1, pp. 100-108, 2010.

[8] G. Ahuja and K. Pathak, "Porous carriers for controlled/ modulated drug delivery," Indian Journal of Pharmaceutical Sciences, vol. 71, no. 6, pp. 599-607, 2009.

[9] M. M. Crowley, B. Schroeder, A. Fredersdorf et al., "Physicochemical properties and mechanism of drug release from ethyl cellulose matrix tablets prepared by direct compression and hotmelt extrusion," International Journal of Pharmaceutics, vol. 269, no. 2, pp. 509-522, 2004.

[10] M. Cristina, Z. Aránzazu, and M. L. José, "Critical factors in the release of drugs from sustained release hydrophilic matrices," Journal of Controlled Release, vol. 154, no. 1, pp. 2-19, 2011.

[11] J. Trygg, E. Yildir, R. Kolakovic, N. Sandler, and P. Fardim, "Anionic cellulose beads for drug encapsulation and release," Cellulose, vol. 21, no. 3, pp. 1945-1955, 2014.

[12] V. M. Volkert, D. C. Lerman, N. A. Call, and N. TrosclairLasserre, "An evaluation of resurgence during treatment with functional communication training," Journal of Applied Behavior Analysis, vol. 42, no. 1, pp. 145-160, 2009.
[13] M. Gericke, J. Trygg, and P. Fardim, "Functional cellulose beads: preparation, characterization, and applications," Chemical Reviews, vol. 113, no. 7, pp. 4812-4836, 2013.

[14] X. Luo and L. Zhang, "Creation of regenerated cellulose microspheres with diameter ranging from micron to millimeter for chromatography applications," Journal of Chromatography A, vol. 1217, no. 38, pp. 5922-5929, 2010.

[15] J. Zhou, C. Chang, R. Zhang, and L. Zhang, "Hydrogels prepared from unsubstituted cellulose in $\mathrm{NaOH}$ /urea aqueous solution," Macromolecular Bioscience, vol. 7, no. 6, pp. 804-809, 2007.

[16] H.-F. Xia, D.-Q. Lin, and S.-J. Yao, "Preparation and characterization of macroporous cellulose-tungsten carbide composite beads for expanded bed applications," Journal of Chromatography A, vol. 1175, no. 1, pp. 55-62, 2007.

[17] L. K. Voon, S. C. Pang, and S. F. Chin, "Highly porous cellulose beads of controllable sizes derived from regenerated cellulose of printed paper wastes," Materials Letters, vol. 164, pp. 264-266, 2015.

[18] H. Wang, D. Li, and R. Zhang, "Preparation of ultralong cellulose nanofibers and optically transparent nanopapers derived from waste corrugated paper pulp," BioResources, vol. 8, no. 1, pp. 1374-1384, 2013.

[19] Q. Wang, X. Xie, X. Zhang, J. Zhang, and A. Wang, "Preparation and swelling properties of $\mathrm{pH}$-sensitive composite hydrogel beads based on chitosan-g-poly (acrylic acid)/vermiculite and sodium alginate for diclofenac controlled release," International Journal of Biological Macromolecules, vol. 46, no. 3, pp. 356-362, 2010.

[20] G. K. Athira and A. N. Jyothi, "Preparation and characterization of curcumin loaded cassava starch nanoparticles with improved cellular absorption," International Journal of Pharmacy and Pharmaceutical Sciences, vol. 6, no. 10, pp. 171-176, 2014.

[21] S. F. Chin, K. S. Iyer, M. Saunders et al., "Encapsulation and sustained release of curcumin using superparamagnetic silica reservoirs," Chemistry - A European Journal, vol. 15, no. 23, pp. 5661-5665, 2009.

[22] Z. Minpeng and L. Suhong, "The stability of curcumin and drug-loading property of starch microspheres for it," Advances in Biomedical Engineering, vol. 9, pp. 44-47, 2012.

[23] P. Costa and J. M. Sousa Lobo, "Modeling and comparison of dissolution profiles," European Journal of Pharmaceutical Sciences, vol. 13, no. 2, pp. 123-133, 2001.

[24] S. Dash, P. N. Murthy, L. Nath, and P. Chowdhury, "Kinetic modeling on drug release from controlled drug delivery systems," Acta Pol Pharm, vol. 67, no. 3, pp. 217-223, 2010.

[25] A. L. Lewis, M. V. Gonzalez, S. W. Leppard et al., "Doxorubicin eluting beads: effects of drug loading on bead characteristics and drug distribution," Journal of Materials Science: Materials in Medicine, vol. 18, no. 9, pp. 1691-1699, 2007.

[26] J. Siepmann and N. A. Peppas, "Modeling of drug release from delivery systems based on hydroxypropyl methylcellulose (HPMC)," Advanced Drug Delivery Reviews, vol. 64, pp. 163-174, 2012.

[27] N. A. Peppas and B. Narasimhan, "Mathematical models in drug delivery: How modeling has shaped the way we design new drug delivery systems," Journal of Controlled Release, vol. 190, pp. 7581, 2014.

[28] M. H. Shoaib, J. Tazeen, H. A. Merchant, and R. I. Yousuf, "Evaluation of drug release kinetics from ibuprofen matrix tablets using HPMC," Pakistan Journal of Pharmaceutical Sciences, vol. 19, no. 2, pp. 119-124, 2006. 
[29] J. Siepmann and F. Siepmann, "Mathematical modeling of drug delivery," International Journal of Pharmaceutics, vol. 364, no. 2, pp. 328-343, 2008. 

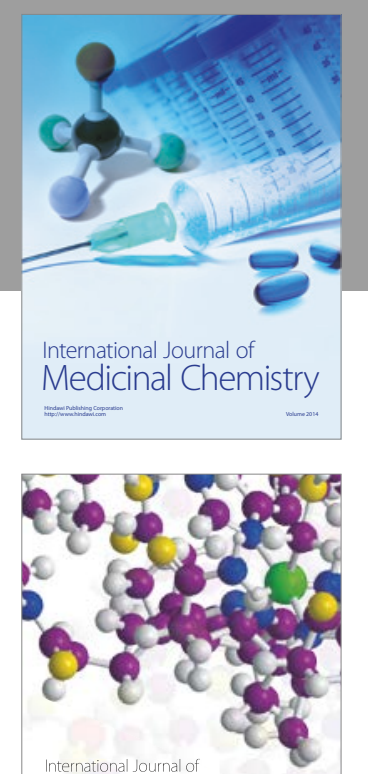

Carbohydrate Chemistry

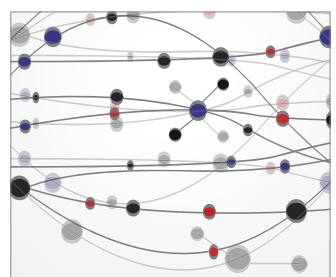

The Scientific World Journal
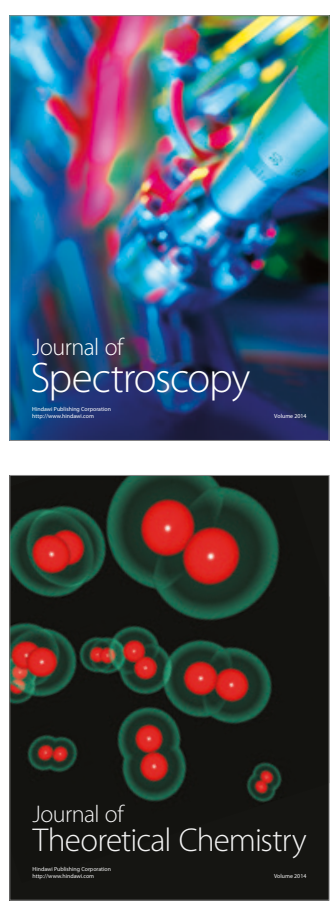
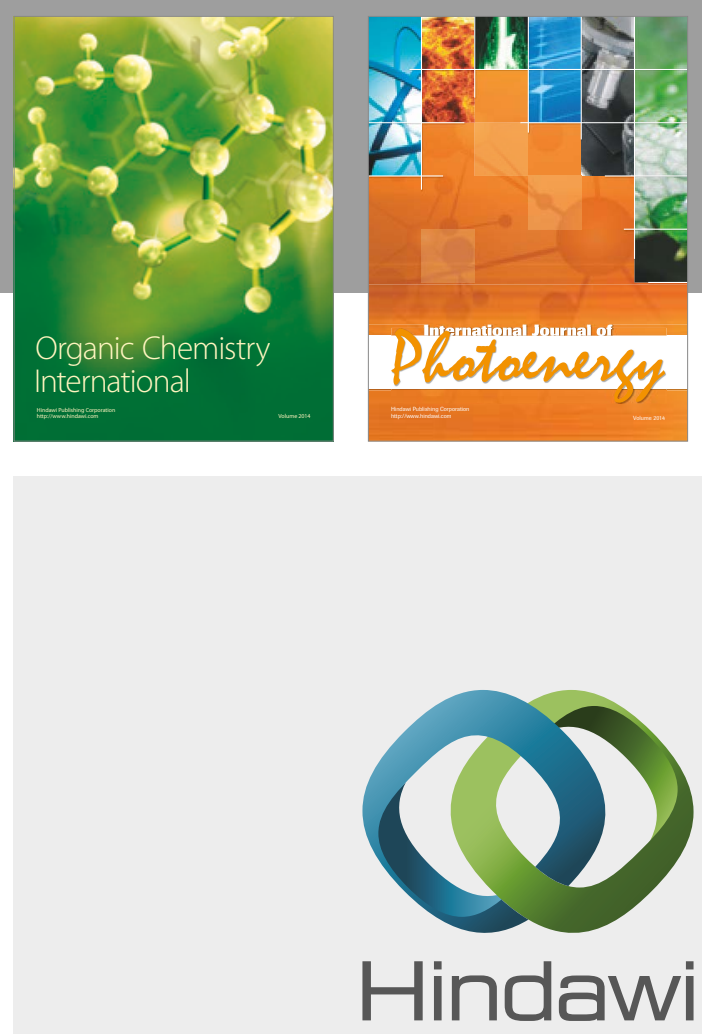

Submit your manuscripts at

https://www.hindawi.com

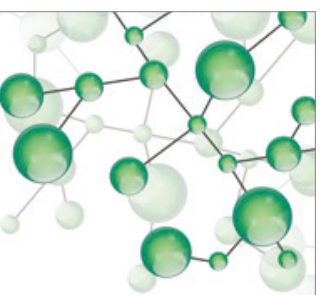

International Journal of

Inorganic Chemistry

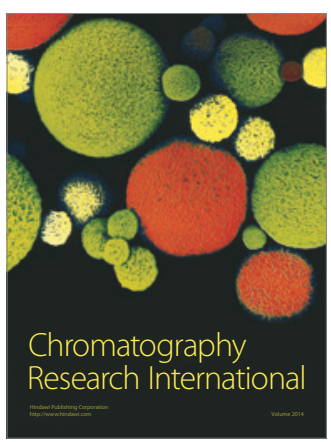

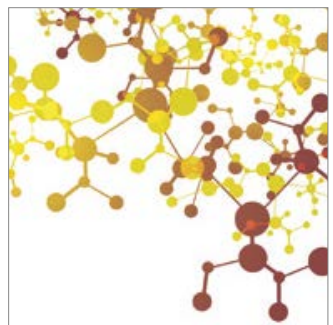

Applied Chemistry
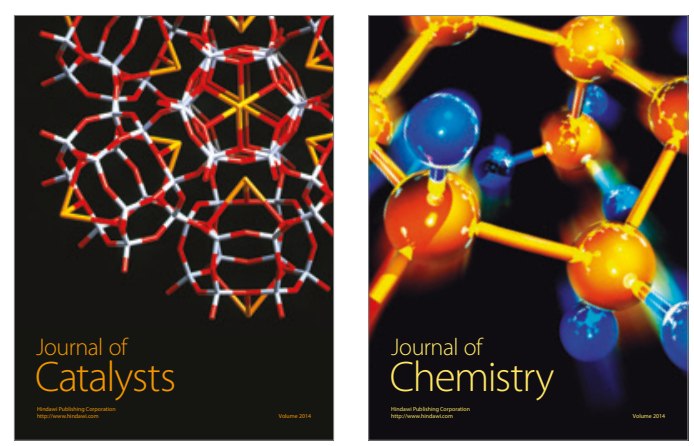
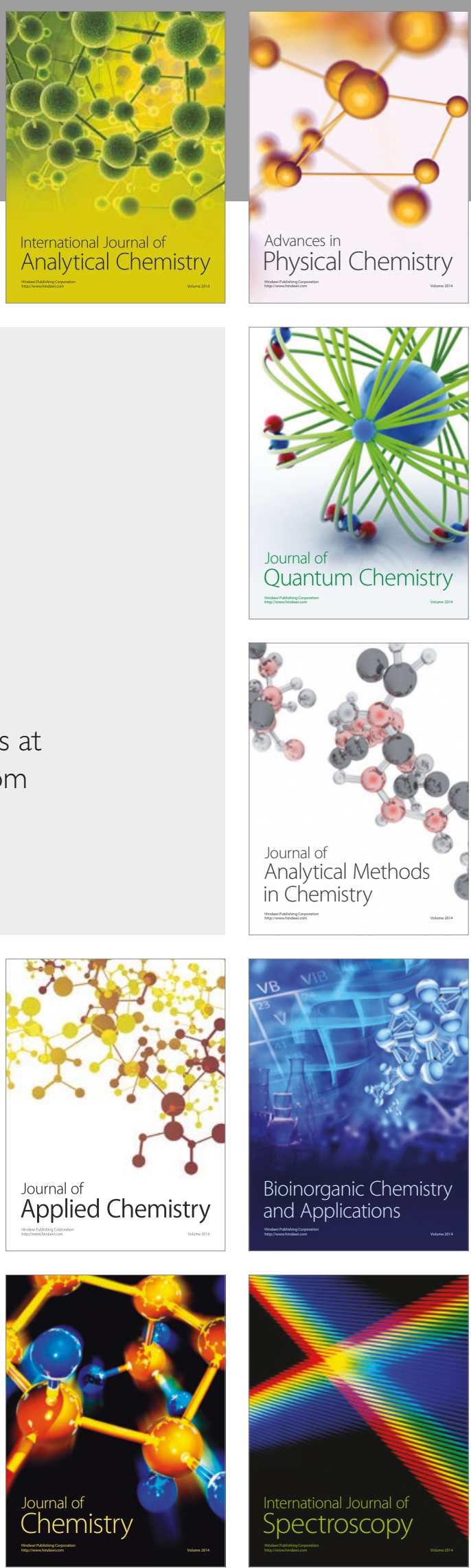